\title{
INQUIRY-BASED BIOMEDICAL SIGNAL PROCESSING LABORATORY: FROM PRACTICE TO SIMULATION
}

\author{
Alan J. Macy \\ BIOPAC Systems, Inc., 42 Aero Camino, Goleta, CA 93117 (805) 685-0066 www.biopac.com \\ Presented at the IEEE EMBS 25th International Conference, Cancun, Mexico, 2003
}

\begin{abstract}
The proposed Biomedical Signal Processing Laboratory incorporates several components that enhance its usefulness for inquiry-based learning. The Laboratory orients around the physical construction and testing of a variety of simple signal processing circuit modules, introduced as lessons. The characteristics of each module can be easily determined through measurement with a BIOPAC data acquisition system. Additionally, the system software permits simple comparisons between real-world and simulated signal processing module characteristics. The modules can be combined in a step-by-step fashion to create a variety of sophisticated and functional signal processing systems. Signal processing systems established by the Laboratory provide meaningful outputs from signals sourced from the student's own body. Through the application of an single, easy-to-use data acquisition system and associated software, students can build and test signal processing modules, verify their performance against mathematical simulation using graphical comparisons, combine modules, collect physiological signals sourced from their own bodies and then analyze the results. In the process of collecting data directly from their own bodies, students' curiosity is stimulated and they gain more control over their own learning by being able to test and retest to more fully understand the steps involved in scientific inquiry.
\end{abstract}

The fusion of practical experience and computer simulation in the context of the Biomedical engineering laboratory is important and meaningful for the student. Buttressing this fusion with the application of a modular, inquiry-based educational approach can result in a very effective learning environment that reflects real-world practice.

Inquiry-based learning is a student-centered, active learning approach focused on questioning, critical thinking and problem solving. An old adage states: "Tell me and I forget, show me and I remember, involve me and I understand." The last part of this statement is the essence of inquiry-based learning. Inquiry-based education is characterized by a learning environment structured to create opportunities for students to be engaged in active learning based upon their own questions. Involvement in learning implies the student possesses capabilities and perceptions that permit the student to look for solutions to problems during the course of acquiring new knowledge.

When presented with the challenge of teaching the fundamentals of signal processing, as they relate to the field of Biomedical Engineering, it's important to apply the concept that inquiry implies involvement that leads to understanding. The proposed Biomedical Signal Processing Laboratory incorporates several components enhancing its usefulness for inquiry-based learning. The Laboratory orients around the physical construction and testing of a variety of simple signal processing circuit modules, each introduced as a lesson. The characteristics of each module can be easily determined through measurement with a BIOPAC MP30 data acquisition system and associated software. The Laboratory permits simple and compelling comparisons between real-world and simulated signal processing module characteristics.

The signal processing circuit modules can be combined in a step-by-step fashion to create a variety of sophisticated and functional signal processing systems. A signal processing system proposed by this Laboratory incorporates the student's own body as the signal source (electrical signal from the heartdetected via skin surface potentials) and provides useful outputs (clinical ECG, hum rejection

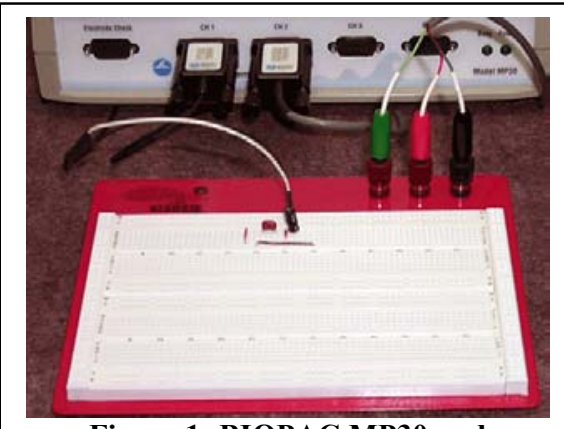

Figure 1: BIOPAC MP30 and SS39L Breadboard and R-wave detector). Through the application of a single, easy-to-use data acquisition system and associated software, students can build and test signal processing modules, verify them against mathematical simulation via graphical comparison, combine modules, and then perform measurements on physiological signals sourced from their own bodies. 
Signal processing circuit modules are introduced sequentially to students as lessons. Students build the modules on a breadboard, evaluate the circuit module characteristics and compare results to mathematical simulation using the BIOPAC MP30 data acquisition system and associated software. The MP30 unit is used to perform multi-channel measurements and supply isolated power to the breadboard. Students use the identical Laboratory software for data collection and simulation, thus reducing the amount of time the teacher needs to spend on training. The "building-block" nature of the signal processing circuit modules encourages students to think of novel connection topologies between the various modules. The requirement that students build a real processing circuit and then compare it to simulation helps create an inquirybased learning environment.

Finally, when students collect data directly from their own bodies, the process stimulates their curiosity and gives them more control over their own learning by allowing them to test and retest to more fully understand the steps involved in scientific inquiry.

The Biomedical Signal Processing Laboratory has the following characteristics:

1) The Laboratory is modular. Signal processing circuit modules can be combined in a large variety of ways to build a number of different real-world biomedical signal processing systems, such as processors for signals originating from the heart (ECG), muscles (EMG), eyes (EOG), stomach (EGG) and brain (EEG).

2) The software application can be used to collect and analyze real data from signal processing circuit modules and perform mathematical simulation. Comparisons between collected and simulated data can be performed in real time and in a graphical manner.

3) The signal processing circuit modules are fundamental processors, largely orthogonal in practical operation. Depending on how modules are combined and modified, systems using similar modules can perform considerably different physiological processing operations. For example, a detector topology for R-wave detection in the ECG becomes (with slight modification) an Alpha wave indicator when recording the EEG.

4) Signal processing circuit modules are introduced sequentially as lessons, allowing students to grasp the performance of each before proceeding to the following module. This process establishes a strong foundation for students to create processing systems of their own design.

The Laboratory introduces eight fundamental signal processing circuit modules (lessons) to the student, as shown in Figures 2-9 and combined in Fig. 13.

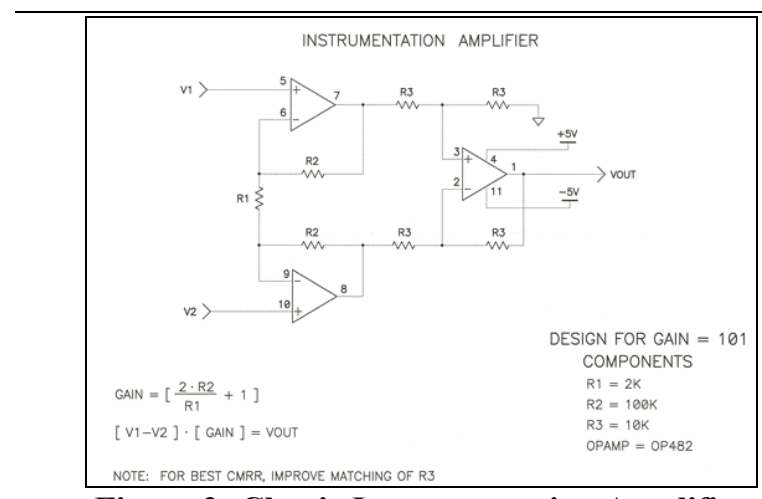

Figure 2: Classic Instrumentation Amplifier

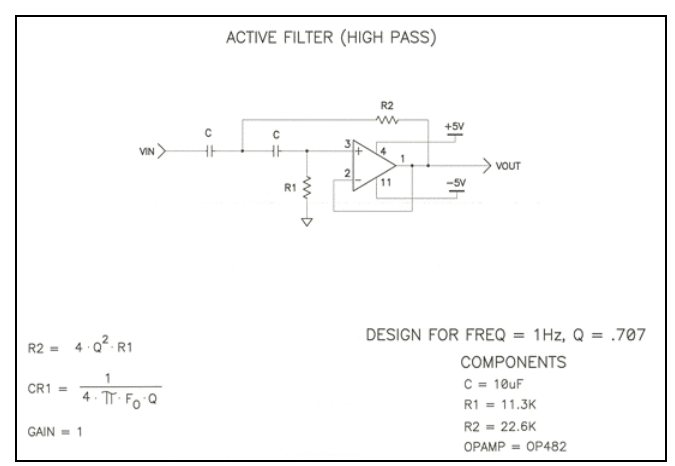

Figure 3: High Pass Filter

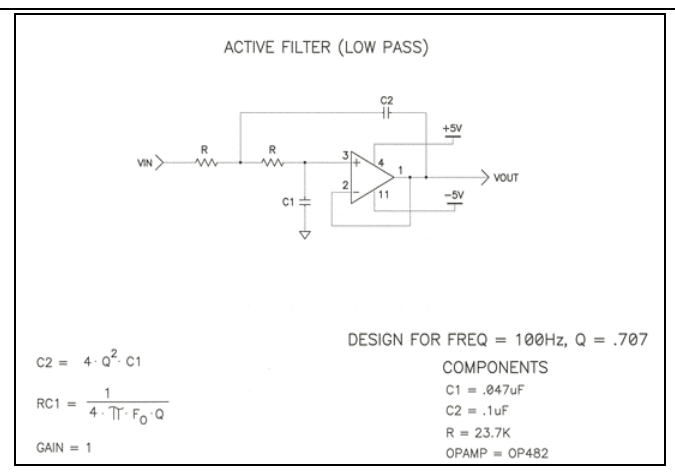

Figure 4: Low Pass Filter

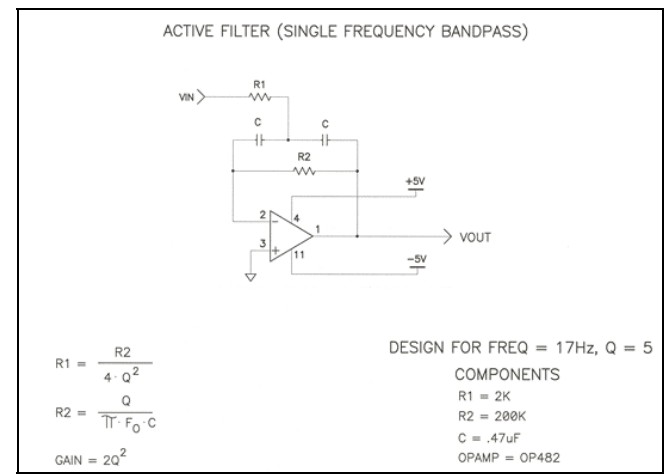

Figure 5: Single Frequency Band Pass Filter 


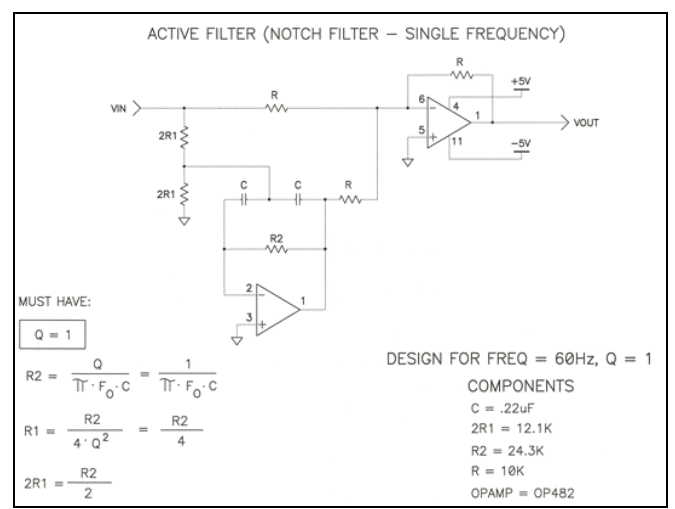

Figure 6: Single Frequency Notch Filter

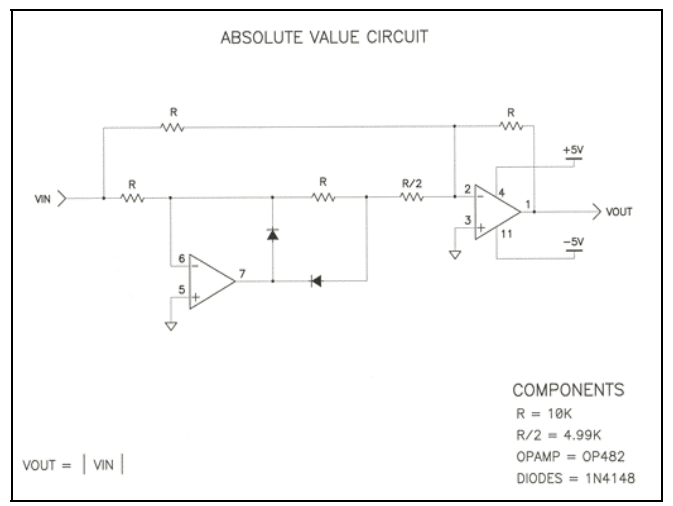

Figure 7: Absolute Value Converter

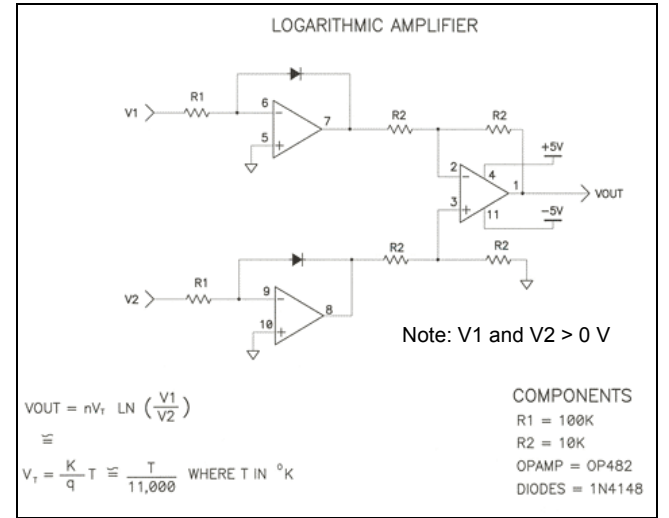

Figure 8: Logarithmic Converter

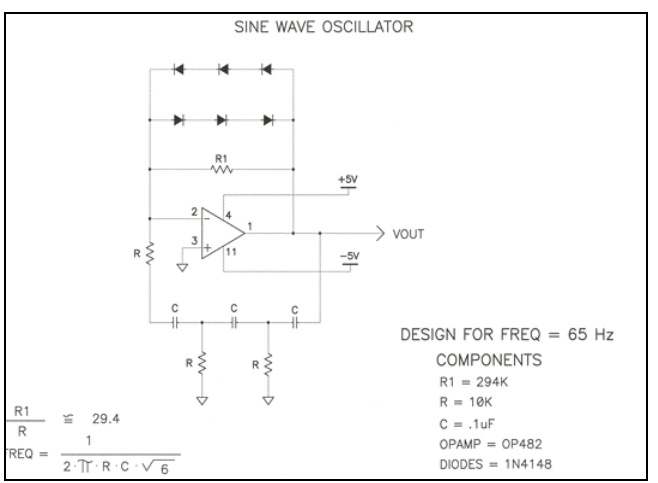

Figure 9: Sine Wave Oscillator
Each signal processing circuit module can be directly emulated (simulated) in software. All the physical biquad filters (low pass, high pass, band pass, notch) are implemented in software as $2^{\text {nd }}$ order IIR filters, operable in real time or post-processing.

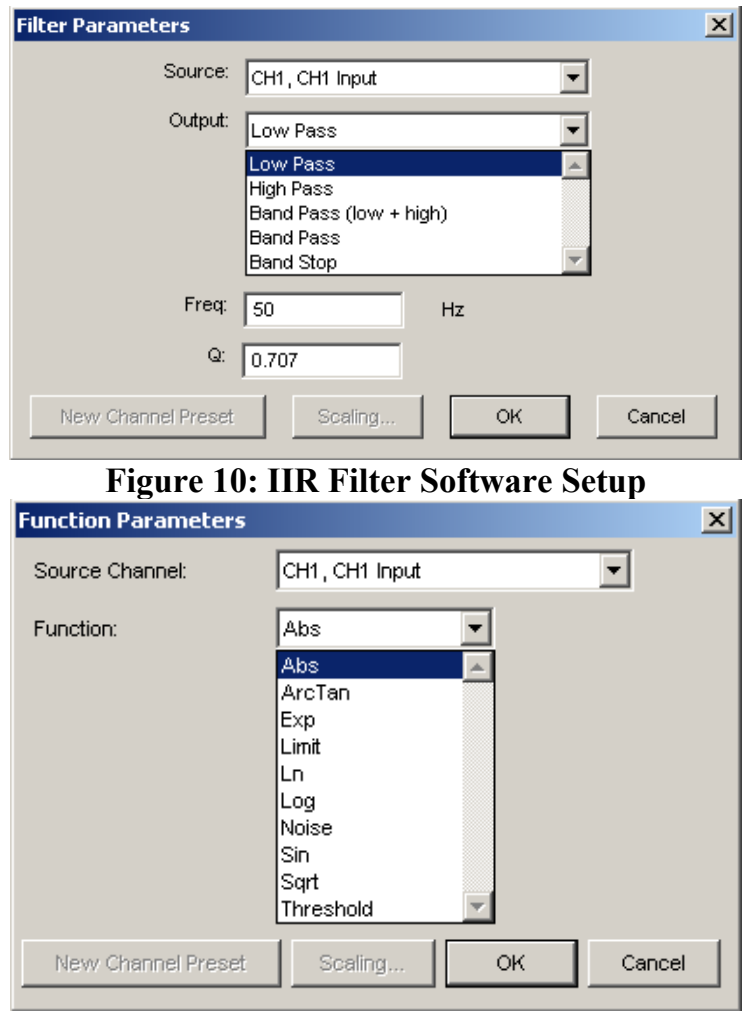

Figure 11: Function Processor Software Setup
Equivalent simulations are available for non-linear signal processing circuits, such as the Absolute Value and Logarithmic Converters.

The software includes an "Equation Generator" which can be used to combine the inputs and outputs of various simulated signal processing modules, much like a physical electrical connection on the breadboard. The Equation Generator also permits the creation of simulated sources like sine, square or arbitrary waves.

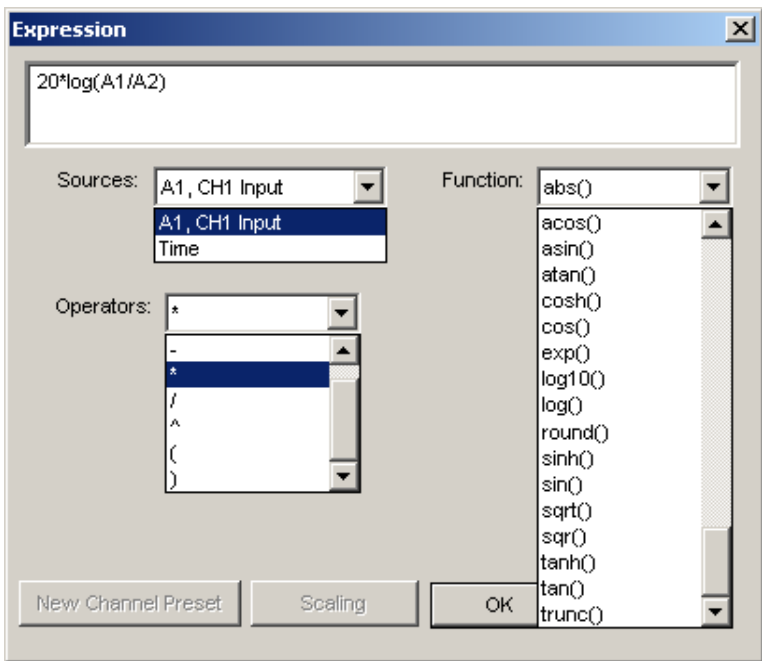

Figure 12: Equation Generator Software Setup 
Finally, the Laboratory creates a simple environment for students to compare actual collected data with data provided by an equivalent mathematical simulation. Such comparisons are typically performed graphically in real time, so students come away with a powerful impression of the utility of simulation as an engineering tool.

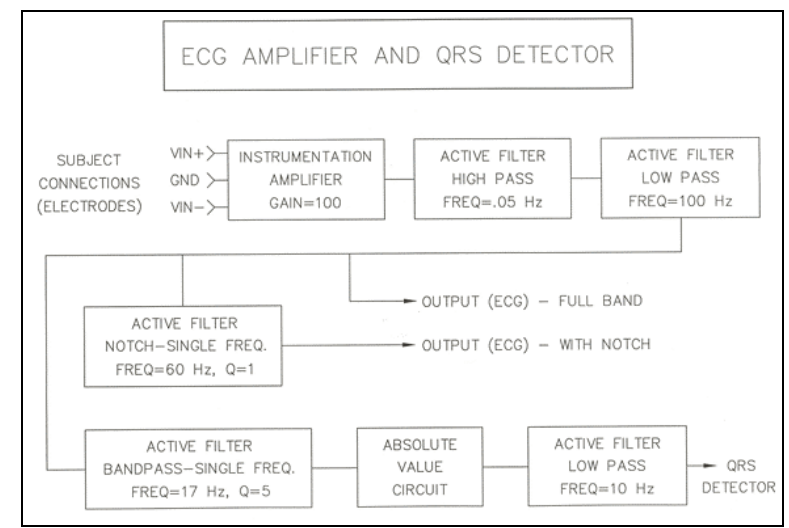

Figure 13: ECG Signal Processor

The results of a simulation that identifies the nature of the signal at the output of each processing circuit in the ECG signal processor (as indicated in Figure 13) are illustrated below (Figure 14). The data shown at the output of each stage is presented in the same form as when the student performs the actual measurement at the signal processing circuit output.

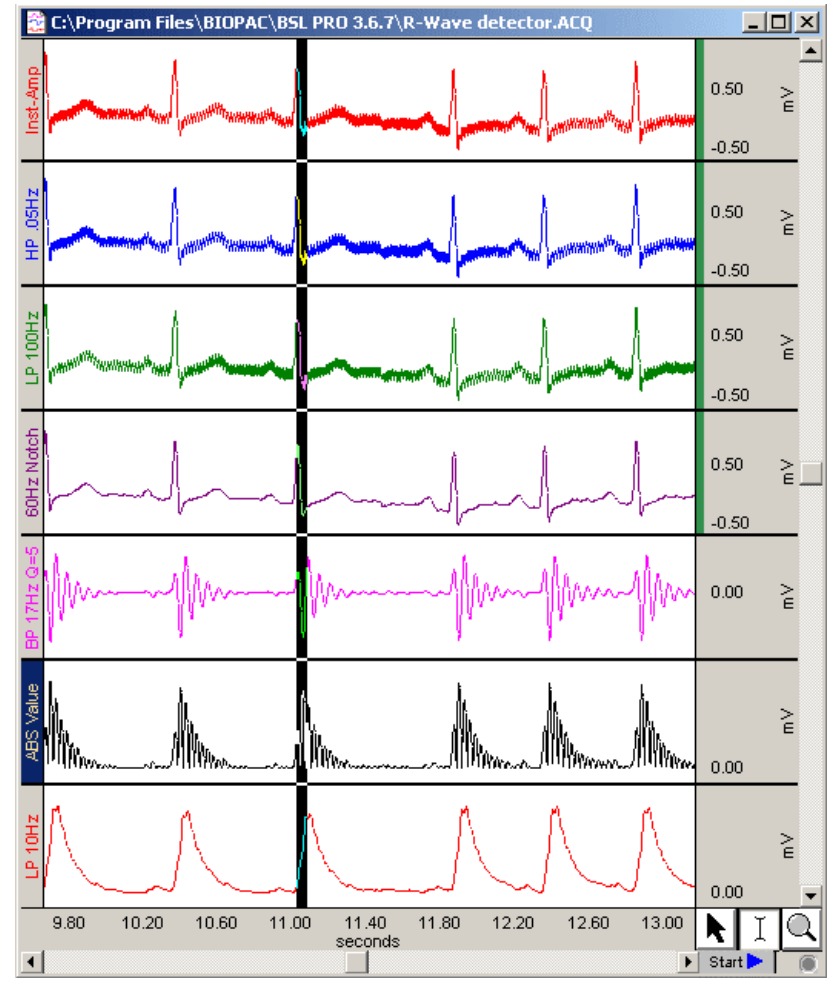

Figure 14: Compare Actual to Simulated Results

Before any lesson, the instructor can set up the associated Laboratory software for the lesson using a Template. Templates are used to pre-configure the MP30 data acquisition system for a particular lesson.
For example, Templates can be used to configure three of the MP30 input channels as single-ended oscilloscope inputs, with the fourth performing a low-level differential voltage measurement. Templates are used to set up acquisition modes in the system, such as: Sampling rate, Acquisition length, and Number of channels. Templates can also be used to reflect laboratory instructions and procedures to the student. Furthermore, for advanced teaching applications, Templates can be used to easily include additional real and simulated processing modules into the Laboratory. Templates are optional, but their use permits quick and global initializations of all the data acquisition systems in the laboratory.

The proposed Biomedical Signal Processing Laboratory can aid in the transition process from instructor-dictated to student-driven, inquiry-based learning. Lesson by lesson, fundamental signal processing modules are introduced to students, requiring them to build, test and simulate. This process viscerally engages the student in an inquirybased learning environment. The modular, "building block" nature of the Laboratory helps develop a strong foundation for additional learning. Finally, the specialized teaching software associated with the BIOPAC MP30 data acquisition system permits students to encounter the laboratory hurdles of proper amplification, signal calibration, scaling and unit assignment, data digitization, signal processing and analysis and simulation-without encountering the myriad set up challenges and time loss that conventional equipment can present.

\section{REFERENCES}

Enderle J., Kelso D., Ropella K. Preparing Biomedical Engineers for Real-World Problem Solving: Proceedings of the Whitaker Foundation BME Educational Summit, 2000.

Lundmark J. and Salmi, A. Inquiry-based Labs using the BIOPAC Student Lab System: (Results from an NSF-CCLI grant-supported project.) HAPS Proceedings, 2001.

Macy A. Students as Signal Sources in the Biomedical Engineering Laboratory: Proceedings of the IEEE EMBS 23 $3^{\text {rd }}$ International Conference, 2001.

Pflanzer R. Experimental and Applied Physiology: Including BIOPAC Lab Experiments: Sixth Edition. (New York: McGraw-Hill, 1999).

Wood M. Laboratory Textbook of Anatomy and Physiology: Second Edition. (New Jersey: Prentice Hall, 2001). 